\title{
Kinetics Investigation on Mushroom Tyrosinase Inhibition of Proso Millet
}

\author{
Wen-Ying Huang $\mathbb{D},{ }^{1}$ Hui-Ju Chen $\mathbb{D}^{2}{ }^{2}$ Chia-Ching Lin, ${ }^{1}$ \\ Chin-Shuh Chen, ${ }^{2}$ and Yung-Sheng Lin (iD $^{3}$ \\ ${ }^{1}$ Department of Applied Cosmetology, Hung-Kuang University, Taichung, Taiwan \\ ${ }^{2}$ Department of Food Science and Biotechnology, National Chung Hsing University, Taichung, Taiwan \\ ${ }^{3}$ Department of Chemical Engineering, National United University, Miaoli City, Taiwan
}

Correspondence should be addressed to Yung-Sheng Lin; linys@nuu.edu.tw

Received 14 October 2017; Accepted 16 January 2018; Published 12 February 2018

Academic Editor: Manuela Curcio

Copyright (C) 2018 Wen-Ying Huang et al. This is an open access article distributed under the Creative Commons Attribution License, which permits unrestricted use, distribution, and reproduction in any medium, provided the original work is properly cited.

Proso millet (Panicum miliaceum) is rich in nutritive components and is widely used as a human food, feed and forage for animals, and fuel. This study investigated the effect of a proso millet extract on the inhibition of tyrosinase, a key enzyme in melanogenesis. High performance liquid chromatography analysis indicated that the proso millet extract contained phenolic tyrosinase inhibitors, such as syringic acid, $p$-coumaric acid, and ferulic acid. The extract had an $\mathrm{IC}_{50}$ for inhibition of tyrosinase activity of $14.02 \mathrm{mg} / \mathrm{mL}$. A Lineweaver-Burk double reciprocal plot showed that the proso millet extract functioned as a mixed competitive and noncompetitive inhibitor. Proso millet has potential as a tyrosinase inhibitor that may have applications in the cosmetics industry.

\section{Introduction}

Proso millet (Panicum miliaceum L.) was first domesticated in East Asia more than 10,000 years ago and is now one of the world's most important and ancient domesticated crops [1]. It has very short growing season (10 weeks) and, aside from wheat and barley, is the longest-used summer cereal crop [2]. Proso millet is used as a human food, feed and forage for livestock, and fuel [3].

There have been many studies of proso millet due to its economic importance. For example, some studies have proposed morphological and molecular analysis to identify proso millet [4-6]. Other studies have examined germination [7] and growth $[8,9]$. Two recent publications reviewed the agronomic characteristics of proso millet $[10,11]$.

Proso millet is a rich source of protein, minerals, and vitamins, and many studies have characterized its biomolecular components. The protein content of proso millet is about $12 \%$ by dry weight, and the protein quality (essential amino acid index) is about $51 \%$ [2]. The major biomolecular components of extracted aromatic hydrocarbons and ethers are miliacin, $\alpha$-amyrin methyl ether, and pentacyclic triterpene methyl ethers, and the relative abundance is miliacin nearly $90 \%$ [1].

Some previous studies have proposed biomedical applications for proso millet. In particular, the protein of proso millet may prevent liver injury induced by D-galactosamine [12] and reduce the plasma concentration of high-density lipoprotein [13]. Proso millet is also a gluten-free grain and is an acceptable food for individuals with coeliac disease or gluten sensitive enthesopathy [14]. Proso millet may also prevent hair loss induced by cisplatin-based chemotherapies [15]. Proso millet affects adipocyte differentiation and downregulates adipogenic genes and fatty acid accumulation in adipocytes [16].

There is increasing interest in the use of plant natural products for inhibition of melanogenesis $[17,18]$. However, little is known about the effect of proso millet on tyrosinase activity. The objective of this study was to determine effect of a proso millet extract on the kinetics of tyrosinase activity as a preliminary assessment for its use in cosmetic applications. 


\section{Materials and Methods}

2.1. Materials. Acetic acid was from Panreac (Barcelona, Spain), acetonitrile and disodium hydrogen phosphate were from J. T. Baker (Phillipsburg, NJ, USA), ferulic acid was from ChromaDEX (Irvine, California), gallic acid was from Alfa Aesar (Ward Hill, MA, USA), kojic acid and (s)-2-amino3-(3,4-dihydroxyphenyl) propanoic acid (L-dopa) were from Acros (New Jersey, USA), and methyl paraben was from Supelco (Bellefonte, USA). Mushroom tyrosinase, caffeic acid, $p$-coumaric acid, and sodium dihydrogen phosphate were from Sigma-Aldrich (Saint Louis, MO, USA). Syringic acid was from MP Biomedicals (Santa Ana, California, USA), and proso millet (milletGen ${ }^{\circledR}$ ) was from Healthmate Co. Ltd. (Changhua, Taiwan).

2.2. Preparation of Proso Millet Extract. One gram of proso millet and $9 \mathrm{~mL}$ of deionized water were mixed and sonicated in an ultrasonic bath for $30 \mathrm{~min}$. The supernatant was collected after centrifugation and then passed through a filter with a $0.45 \mu \mathrm{m}$ pore size. A $0.1 \mathrm{mg} / \mathrm{mL}$ methyl paraben solution was dissolved in $70 \%$ methanol and passed through a $0.45 \mu \mathrm{m}$ filter, and the filtrate was utilized as an internal standard in high performance liquid chromatography (HPLC) analysis. For HPLC analysis, the proso millet solution was prepared by mixing $180 \mu \mathrm{L}$ of the proso millet supernatant with $10 \mu \mathrm{L}$ of the internal standard solution. For tyrosinase inhibition, the proso millet solution was diluted with deionized water into a series of solutions $(6.25,12.5,17.5,20$, and $25 \mathrm{mg} / \mathrm{mL}$ ).

2.3. Calibration and Validation. Standard solutions of syringic acid, $p$-coumaric acid, and ferulic acid were prepared at $0.5 \mathrm{mg} / \mathrm{mL}$ in methanol, and series of diluted standards were prepared for HPLC calibration curves (syringic acid: $0.45,0.563,0.9,1.125$, and $2.25 \mu \mathrm{g} / \mathrm{mL}$; $p$-coumaric acid: 1.5 , $1.8,2.25,3$, and $4.5 \mu \mathrm{g} / \mathrm{mL}$; ferulic acid: $0.75,0.9,1.125,1.5$, 2.25 , and $4.5 \mu \mathrm{g} / \mathrm{mL}$ ) with internal standard solution $(18: 1)$. The criterion of a signal to noise $(\mathrm{S} / \mathrm{N})$ ratio of at least $3: 1$ was used as the detection limit. Recovery of a standard marker was defined as the ratio of the detected amount to the added amount.

2.4. HPLC Analysis. The HPLC system (Agilent 1200 Infinity Series, Agilent, USA) had a reverse-phase column (Cosmosil 5C18-AR II, $5 \mu \mathrm{m}, 25 \mathrm{~cm} \times 4.6 \mathrm{~mm}$ ID, Nacalai Tesque, Kyoto, Japan). The mobile phase consisted of a mixture of $0.5 \%$ acetic acid and $80 \%$ acetonitrile. The percentage of $0.5 \%$ acetic acid in the mobile phase was $100 \%$ at $0 \mathrm{~min}, 80 \%$ at $0-10 \mathrm{~min}, 70 \%$ at $10-20 \mathrm{~min}, 40 \%$ at $20-30 \mathrm{~min}$, and $0 \%$ at $30-40 \mathrm{~min}$. The flow rate was $0.8 \mathrm{~mL} / \mathrm{min}$ and absorbance was measured at $280 \mathrm{~nm}$.

2.5. Tyrosinase Inhibition. Based on a previous report [19], $40 \mu \mathrm{L}$ of proso millet solution ( 6.25 to $25 \mathrm{mg} / \mathrm{mL}$ ), $40 \mu \mathrm{L}$ of tyrosinase solution $(5.544 \mu \mathrm{g} / \mathrm{mL}$ or $20 \mathrm{U} / \mathrm{mL})$, and $120 \mu \mathrm{L}$ of $5 \mathrm{mM}$ L-dopa solution were loaded into the wells of a 96-well plate at $37^{\circ} \mathrm{C}$. After $30 \mathrm{~min}$, the absorbance was measured at $475 \mathrm{~nm}$. Kojic acid ( 0.035 to $0.2 \mathrm{mg} / \mathrm{mL}$ ) was the positive control, and deionized water was the blank control. The percent tyrosinase inhibition was defined as $100 \% \times$ $\Delta \mathrm{OD}_{\text {sample }} / \Delta \mathrm{OD}_{\text {control }}$, where $\Delta \mathrm{OD}$ is the absorbance change at $475 \mathrm{~nm}$. The half inhibitory concentration $\left(\mathrm{IC}_{50}\right)$ was obtained by regression analysis.

2.6. Kinetic Properties. The Lineweaver-Burk equation can be derived from the Michaelis-Menten equation:

$$
\frac{1}{V}=\frac{K_{m}}{V_{\max }} \times \frac{1}{[S]}+\frac{1}{V_{\max }},
$$

where $V, V_{\max }, K_{m}$, and $[S]$ are reaction rate, maximum reaction rate, Michaelis-Menten constant, and substrate concentration, respectively. The $V_{\max }$ and $K_{m}$ can be obtained by the $y$-intercept $\left(1 / V_{\max }\right)$ and $x$-intercept $\left(-1 / K_{m}\right)$ from a plot of $1 / V$ versus $1 /[S]$ (Lineweaver-Burk plot).

Before constructing the Lineweaver-Burk plot, the effect of the proso millet extract concentration on tyrosinase activity was determined. The concentration of L-dopa (substrate) was set $1.25 \mathrm{mM}$, and $120 \mu \mathrm{L}$ in a sodium phosphate buffer (pH 6.8) was used for testing in a 96-well plate. A $40 \mu \mathrm{L}$ solution of proso millet extract ( 0 to $7 \mathrm{mg} / \mathrm{mL}$ ) and a $40 \mu \mathrm{L}$ solution of tyrosinase $(2.772$ to $11.088 \mu \mathrm{g} / \mathrm{mL})$ were mixed with L-dopa.

For measurement of tyrosinase inhibition, a $40 \mu \mathrm{L}$ solution of solution ( 0 to $7 \mathrm{mg} / \mathrm{mL}$ ) and a $40 \mu \mathrm{L}$ solution of tyrosinase $(5.544 \mu \mathrm{g} / \mathrm{mL})$ were added to each well of a 96well plate. Then a $120 \mu \mathrm{L}$ of an L-dopa solution (0.0625 to $0.25 \mathrm{mM}$ ) in a sodium phosphate buffer ( $\mathrm{pH}$ 6.8) was added to initiate the reaction. A Lineweaver-Burk plot was used to assess the effect of proso millet extract on tyrosinase kinetics.

\section{Results and Discussion}

3.1. HPLC Analysis. Figure 1 shows a representative HPLC chromatogram of the proso millet extract and the internal standard, methyl paraben, which is a stable compound and separated from other peaks. The identified bioactive constituents are syringic acid, $p$-coumaric acid, and ferulic acid. The detection limit was $0.045 \mu \mathrm{g} / \mathrm{mL}$ for syringic acid, $0.078 \mu \mathrm{g} / \mathrm{mL}$ for $p$-coumaric acid, and $0.075 \mu \mathrm{g} / \mathrm{mL}$ for ferulic acid. The recoveries were $97.55 \%$ for syringic acid, $97.01 \%$ for $p$-coumaric acid, and $96.77 \%$ for ferulic acid. The calibration curves of standards were as follows: $y=264.31 x-0.0153, R^{2}=$ 0.9962 for syringic acid; $y=255.56 x+0.0562, R^{2}=0.9991$ for $p$-coumaric acid; $y=188.42 x-0.0123, R^{2}=0.9922$ for ferulic acid. The proso millet extract had $2.81 \mu \mathrm{g} / \mathrm{g}$ syringic acid, $31.88 \mu \mathrm{g} / \mathrm{g} p$-coumaric acid, and $12.52 \mu \mathrm{g} / \mathrm{g}$ ferulic acid.

3.2. Effect of Proso Millet on Tyrosinase Activity. We used kojic acid as a positive control (Figure 2). Figure 3 shows the inhibitory effect of the proso millet extract. These results indicate that kojic acid and proso millet reduced tyrosinase activity in a dose-dependent manner. The $\mathrm{IC}_{50}$ of kojic acid was $0.05 \mathrm{mg} / \mathrm{mL}$ and the $\mathrm{IC}_{50}$ of the proso millet extract was $14.02 \mathrm{mg} / \mathrm{mL}$.

3.3. Kinetics of Tyrosinase Inhibition. Figure 4 shows the relationship between the activity and concentration of tyrosinase 


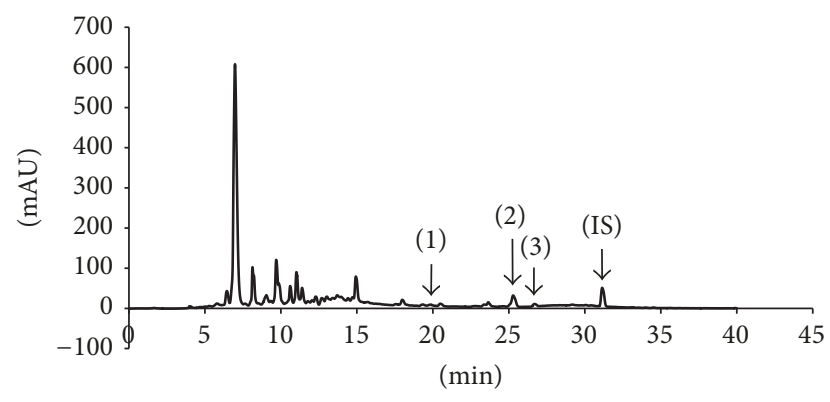

FIgURE 1: Representative HPLC chromatogram of a proso millet extract ((1) syringic acid; (2) p-coumaric acid; (3) ferulic acid) and the internal standard (IS), methyl paraben.

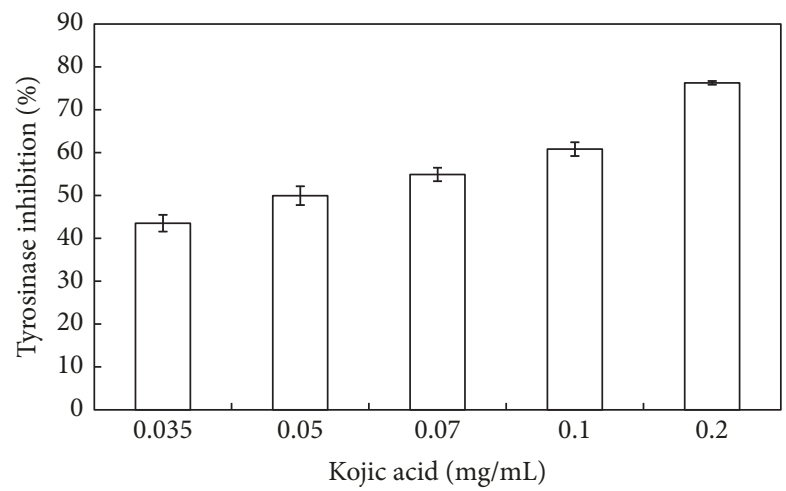

FIGURE 2: Effect of the kojic acid concentration on inhibition of tyrosinase.

in the presence of different concentrations of the proso millet extract when using L-dopa $(1.25 \mathrm{mM})$ as a substrate. These results indicate almost linear relationships between the tyrosinase concentration and activity for each concentration of proso millet extract and that tyrosinase activity decreased as the concentration of the proso millet extract increased, in agreement with the results in Figure 3. This effect may be attributed to the presence of phenolic tyrosinase inhibitors [20-22], such as syringic acid [23], p-coumaric acid [24,25], and ferulic acid [25], which can act as alternative substrates (competitive inhibitors) of tyrosinase [26].

We examined the mechanism of this inhibitory effect using a Lineweaver-Burk double reciprocal plot of reaction rate versus L-dopa concentration with different concentrations of the proso millet extract (Figure 5(a)). The results indicate that the $y$-intercept and $x$-intercept depend on the concentration of the proso millet extract. These changes in the apparent $V_{\max }$ and $K_{m}$ indicate that the proso millet extract inhibited tyrosinase by competitive and noncompetitive mechanisms. Previous research reported that kojic acid and n-acetyl-pentapeptides have similar effects [27], but Vitis vinifera leaf extracts exhibit competitive inhibition [19].

The equilibrium constant (KI) for inhibitor binding with free tyrosinase, determined by linear regression of the apparent $K_{m} / V_{\max }$ versus proso millet extract concentration, was $3.17 \mathrm{mg} / \mathrm{mL}$ (Figure 5(b)). The KI for inhibitor binding with the enzyme-substrate complex [28], determined by linear

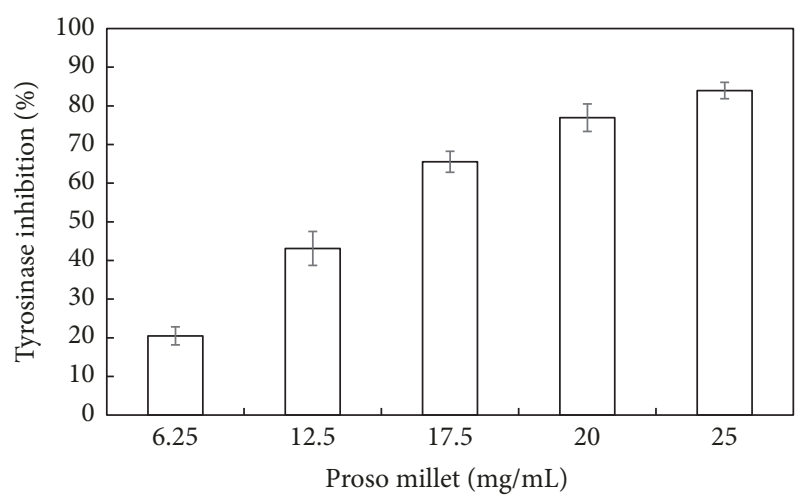

FIGURE 3: Effect of the proso millet extract concentration on the inhibition of tyrosinase.

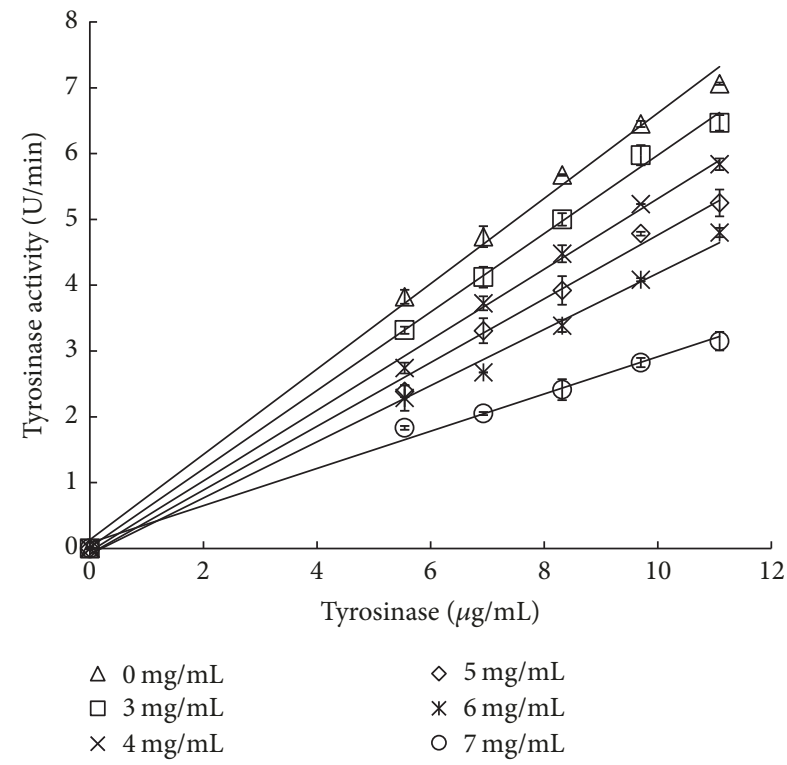

FIGURE 4: Effect of proso millet extract concentration $(0 \sim 7 \mathrm{mg} / \mathrm{mL})$ on the relationship between tyrosinase concentration and activity.

regression of the apparent $1 / V_{\max }$ versus proso millet extract concentration, was $24.28 \mathrm{mg} / \mathrm{mL}$ (Figure 5(c)). The lower the $\mathrm{KI}$ is, the stronger the affinity is. Therefore, proso millet extract has a stronger affinity with free tyrosinase than tyrosinase-L-dopa complex.

\section{Conclusion}

The cosmetics industry is interested in plant natural tyrosinase inhibitors for potential use as inhibitors of melanogenesis. This study investigated the kinetics of tyrosinase inhibition by a proso millet extract. The results indicate that this extract reduced the tyrosinase activity in a dose-dependent manner, with an $\mathrm{IC}_{50}$ of $14.02 \mathrm{mg} / \mathrm{mL}$. A Lineweaver-Burk double reciprocal plot indicated that the proso millet extract functioned as a mixed competitive and noncompetitive inhibitor of tyrosinase. Based on HPLC analysis, the bioactive constituents of the proso millet extract may be phenolics, 


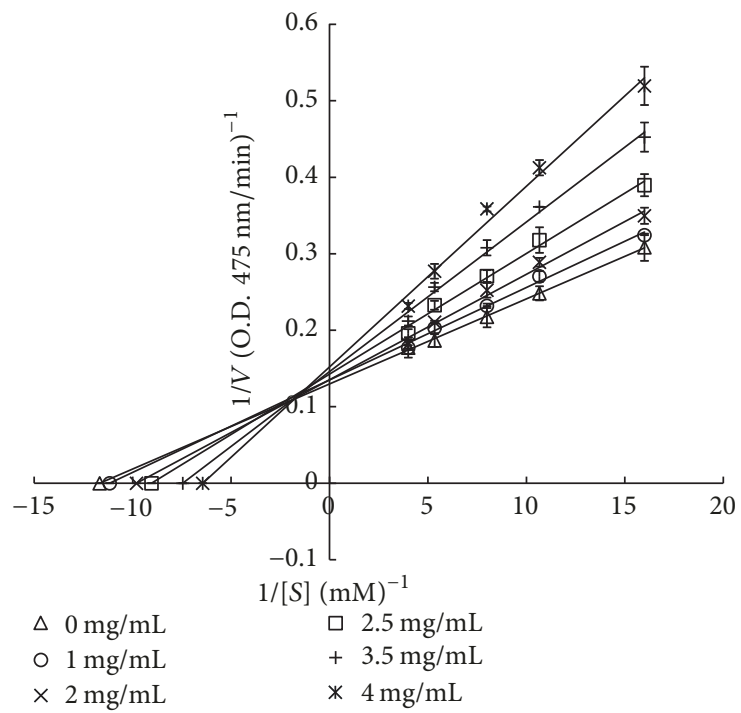

(a)

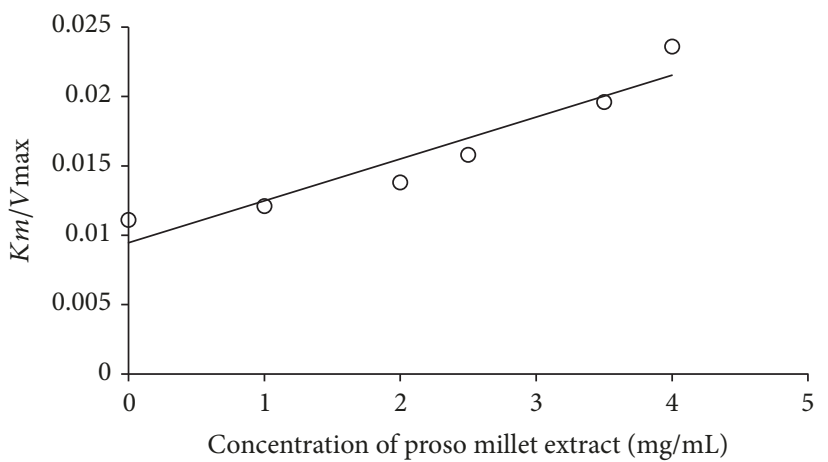

(b)

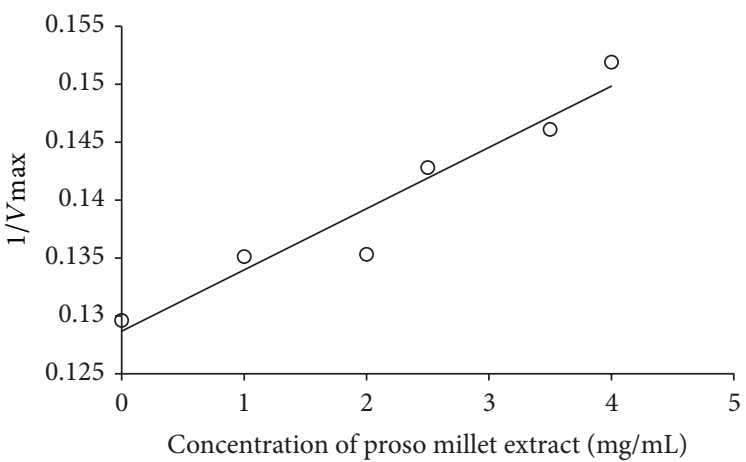

(c)

FIGURE 5: (a) Lineweaver-Burk double reciprocal plot of the effect of proso millet extract concentration (0 4 mg/mL) on tyrosinase activity. (b) Apparent $K_{m} / V_{\max }$ as a function of proso millet extract concentration. (c) Apparent $1 / V_{\max }$ as a function of proso millet extract concentration.

including syringic acid, $p$-coumaric acid, or ferulic acid. These results provide a foundation for the potential use of proso millet as an inhibitor of melanogenesis.

\section{Conflicts of Interest}

The authors declare no conflicts of interest.

\section{Acknowledgments}

The authors are grateful for financial support from the Ministry of Science and Technology, Taiwan, under Contract no. MOST 106-2622-E-239-001-CC3.

\section{References}

[1] H. Lu, J. Zhang, and K.-B. Liu, "Earliest domestication of common millet (Panicum miliaceum) in East Asia extended to 10,000 years ago," Proceedings of the National Acadamy of Sciences of the United States of America, vol. 106, no. 18, pp. 7367$7372,2009$.
[2] J. Kalinova and J. Moudry, "Content and quality of protein in proso millet (Panicum miliaceum L.) varieties," Plant Foods for Human Nutrition, vol. 61, no. 1, pp. 45-49, 2006.

[3] S. L. Kothari, S. Kumar, R. K. Vishnoi, A. Kothari, and K. N. Watanabe, "Applications of biotechnology for improvement of millet crops: Review of progress and future prospects," Plant Biotechnology Journal, vol. 22, no. 2, pp. 81-88, 2005.

[4] S. J. McCanny and P. B. Cavers, "Spread of proso millet (Panicum miliaceum L.) in Ontario, Canada. II. Dispersal by combines," Weed Research, vol. 28, no. 2, pp. 67-72, 1988.

[5] R. Lágler, G. Gyulai, M. Humphreys et al., "Morphological and molecular analysis of common millet (P. miliaceum) cultivars compared to an aDNA sample from the 15th century (Hungary)," Euphytica, vol. 146, no. 1-2, pp. 77-85, 2005.

[6] H. Lu, J. Zhang, N. Wu, K.-B. Liu, D. Xu, and Q. Li, "Phytoliths analysis for the discrimination of Foxtail millet (Setaria italica) and Common millet (Panicum miliaceum)," PLoS ONE, vol. 4, no. 2, Article ID e4448, 2009.

[7] B. Kamkar, A. Koocheki, M. Nassiri Mahallati, and P. Rezvani Moghaddam, "Cardinal temperatures for germination in three millet specieses (Panicum miliaceum, Pennisetum glaucum and Setaria italica)," Asian Journal of Plant Sciences, vol. 5, no. 2, pp. 316-319, 2006. 
[8] M. J. Seghatoleslami, M. Kafi, and E. Majidi, "Effect of drought stress at different growth stages on yield and water use efficiency of five proso millet (Panicum miliaceum l.) genotypes," Pakistan Journal of Botany, vol. 40, no. 4, pp. 1427-1432, 2008.

[9] D. Yoon, S. Oh, B. Lee et al., "Effects of Ridge Width on Growth and Yield of Proso Millet (Panicum miliaceum L.) in PaddyUpland Rotation Field," The Korean Journal of Crop Science, vol. 60, no. 2, pp. 217-223, 2015.

[10] C. Habiyaremye, J. B. Matanguihan, J. D’Alpoim Guedes et al., "Proso millet (Panicum miliaceum L.) and its potential for cultivation in the Pacific Northwest, U.S.: A review," Frontiers in Plant Science, vol. 7, article no. 1961, 2017.

[11] R. Wang, H. V. Hunt, Z. Qiao, L. Wang, and Y. Han, “Diversity and Cultivation of Broomcorn Millet (Panicum miliaceum L.) in China: A Review," Economic Botany, vol. 70, no. 3, pp. 332342, 2016.

[12] N. Nishizawa, D. Sato, Y. Ito et al., "Effects of dietary protein of proso millet on liver injury induced by d-galactosamine in rats," Bioscience, Biotechnology, and Biochemistry, vol. 66, no. 1, pp. 92-96, 2002.

[13] N. Nishizawa and Y. Fudamoto, "The Elevation of Plasma Concentration of High-Density Lipoprotein Cholesterol in Mice Fed with Protein from Proso Millet," Bioscience, Biotechnology, and Biochemistry, vol. 59, no. 2, pp. 333-335, 1995.

[14] E. Aubrecht, M. Horacsek, É. Gelencsér, and E. Dworschak, "Investigation of prolamin content of cereals and different plant seeds," Acta Alimentaria, vol. 27, no. 2, pp. 119-125, 1998.

[15] G. Gardani, R. Cerrone, C. Biella et al., "A case-control study of Panicum Miliaceum in the treatment of cancer chemotherapyinduced alopecia," Minerva Medica, vol. 98, no. 6, pp. 661-664, 2007.

[16] M.-Y. Park, D.-W. Seo, J.-Y. Lee et al., "Effects of Panicum miliaceum L. extract on adipogenic transcription factors and fatty acid accumulation in 3T3-11 adipocytes," Nutrition Research and Practice, vol. 5, no. 3, pp. 192-197, 2011.

[17] C.-C. Tsai, C.-F. Chan, W.-Y. Huang et al., "Applications of Lactobacillus rhamnosus spent culture supernatant in cosmetic antioxidation, whitening and moisture retention applications," Molecules, vol. 18, no. 11, pp. 14161-14171, 2013.

[18] C.-F. Chan, C.-C. Huang, M.-Y. Lee, and Y.-S. Lin, "Fermented broth in tyrosinase- and melanogenesis inhibition," Molecules, vol. 19, no. 9, pp. 13122-13135, 2014.

[19] Y.-S. Lin, H.-J. Chen, J.-P. Huang et al., "Kinetics of tyrosinase inhibitory activity using vitis vinifera leaf extracts," BioMed Research International, vol. 2017, Article ID 5232680, 2017.

[20] C. Gómez-Cordovés, B. Bartolomé, W. Vieira, and V. M. Virador, "Effects of wine phenolics and sorghum tannins on tyrosinase activity and growth of melanoma cells," Journal of Agricultural and Food Chemistry, vol. 49, no. 3, pp. 1620-1624, 2001.

[21] Y.-J. Kim and H. Uyama, "Tyrosinase inhibitors from natural and synthetic sources: Structure, inhibition mechanism and perspective for the future," Cellular and Molecular Life Sciences, vol. 62, no. 15, pp. 1707-1723, 2005.

[22] Z.-P. Zheng, Q. Zhu, C.-L. Fan, H.-Y. Tan, and M. Wang, "Phenolic tyrosinase inhibitors from the stems of Cudrania cochinchinensis," Food \& Function, vol. 2, no. 5, pp. 259-264, 2011.

[23] X. Chen, A. Haniu, T. Kashiwagi et al., "Isolation and identification of tryrosinase-inhibition active compounds from Diplomorpha sikokiana," Academia Journal of Medicinal Plants, vol. 4, no. 8, pp. 10-15, 2016.
[24] J.-Y. Lim, K. Ishiguro, and I. Kubo, “Tyrosinase inhibitory pcoumaric acid from ginseng leaves," Phytotherapy Research, vol. 13, no. 5, pp. 371-375, 1999.

[25] J. W. Lin, H. M. Chiang, Y. C. Lin, and K. C. Wen, "Natural products with skin-whitening effects," Journal of Food and Drug Analysis, vol. 16, no. 2, pp. 1-10, 2008.

[26] S. Passi and M. Nazzaro-Porro, "Molecular basis of substrate and inhibitory specificity of tyrosinase: phenolic compounds," British Journal of Dermatology, vol. 104, no. 6, pp. 659-665, 1981.

[27] C.-Y. Lien, C.-Y. Chen, S.-T. Lai, and C.-F. Chan, "Kinetics of mushroom tyrosinase and melanogenesis inhibition by $\mathrm{N}$-acetyl-pentapeptides," The Scientific World Journal, vol. 2014, Article ID 409783, 9 pages, 2014.

[28] W. C. Liao, W. H. Wu, P. C. Tsai, H. F. Wang, Y. H. Liu, and C. F. Chan, "Kinetics of ergothioneine inhibition of mushroom tyrosinase," Applied Biochemistry and Biotechnology, vol. 166, no. 2, pp. 259-267, 2012. 

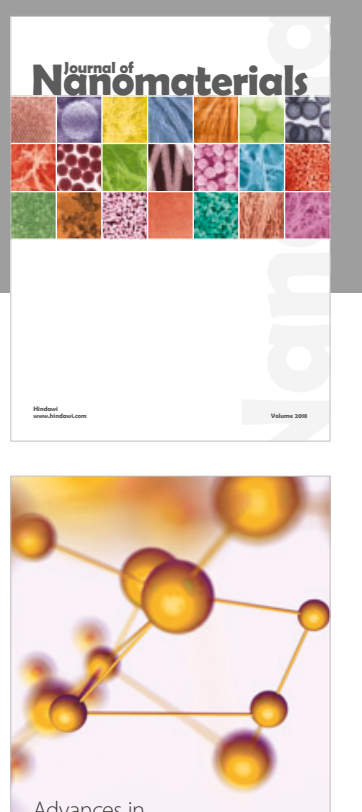

Physical Chemistry
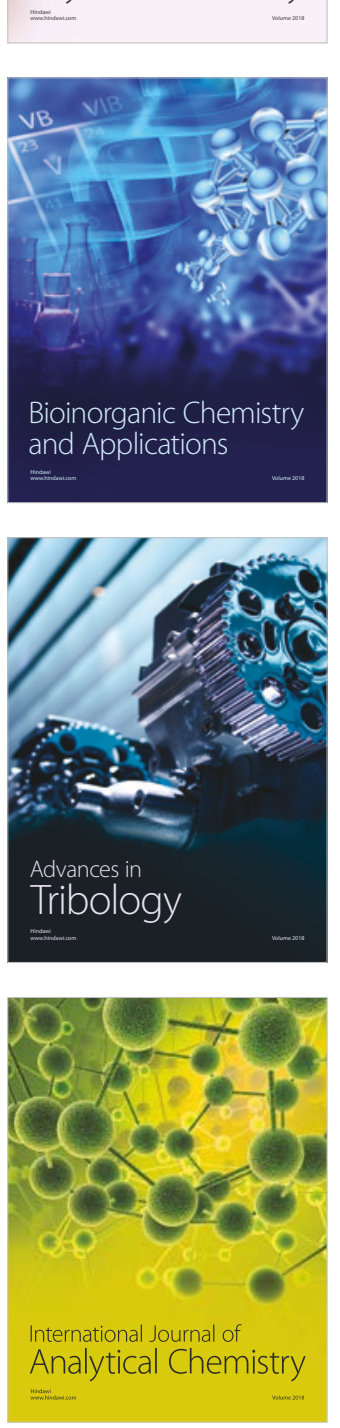

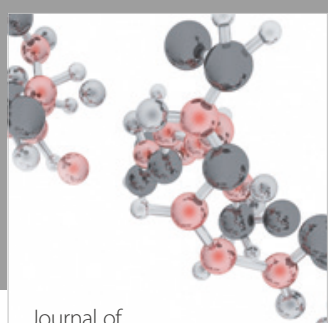

Analytical Methods

in Chemistry

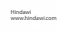

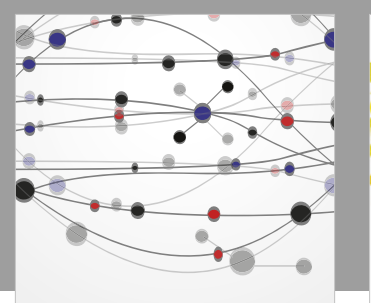

The Scientific World Journal

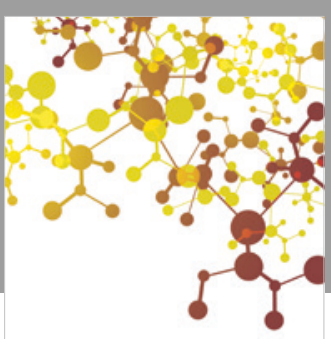

Journal of

Applied Chemistry
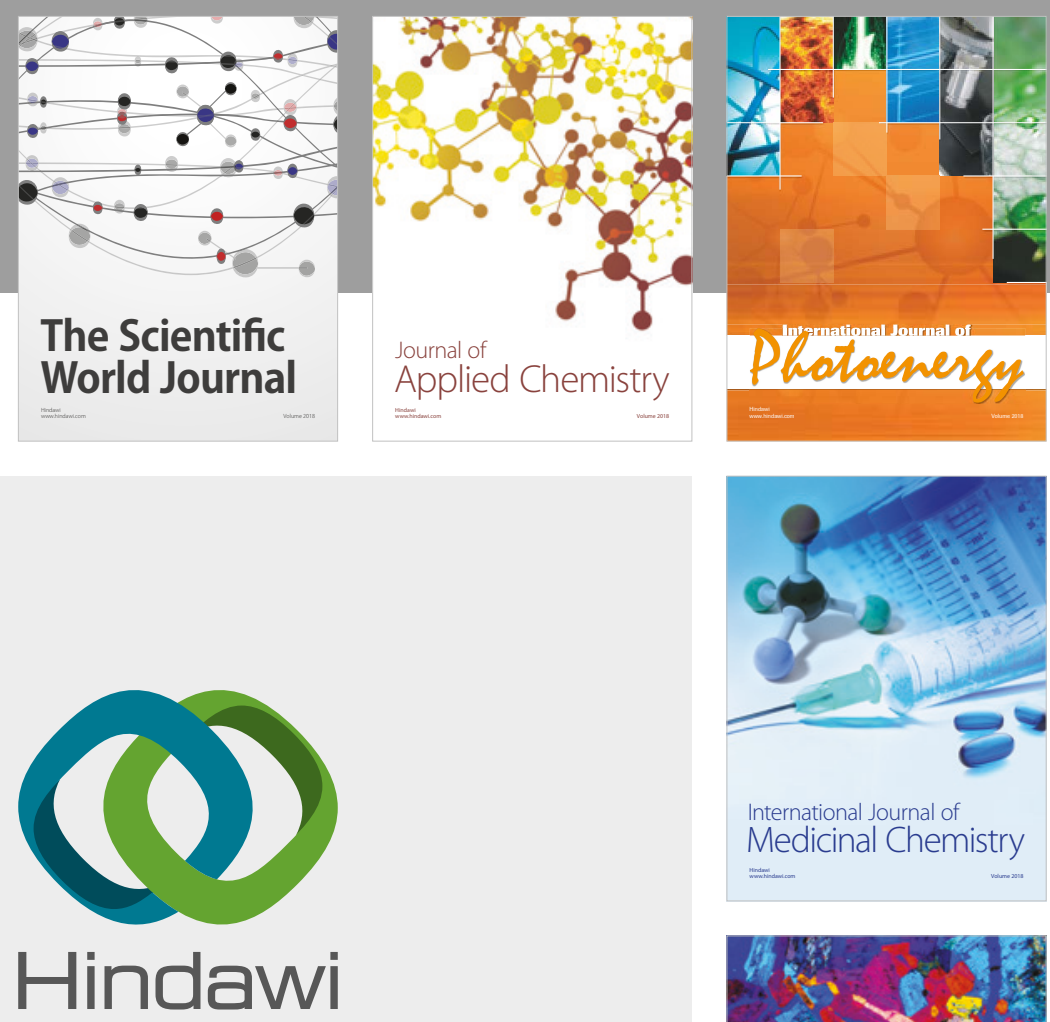

Submit your manuscripts at

www.hindawi.com
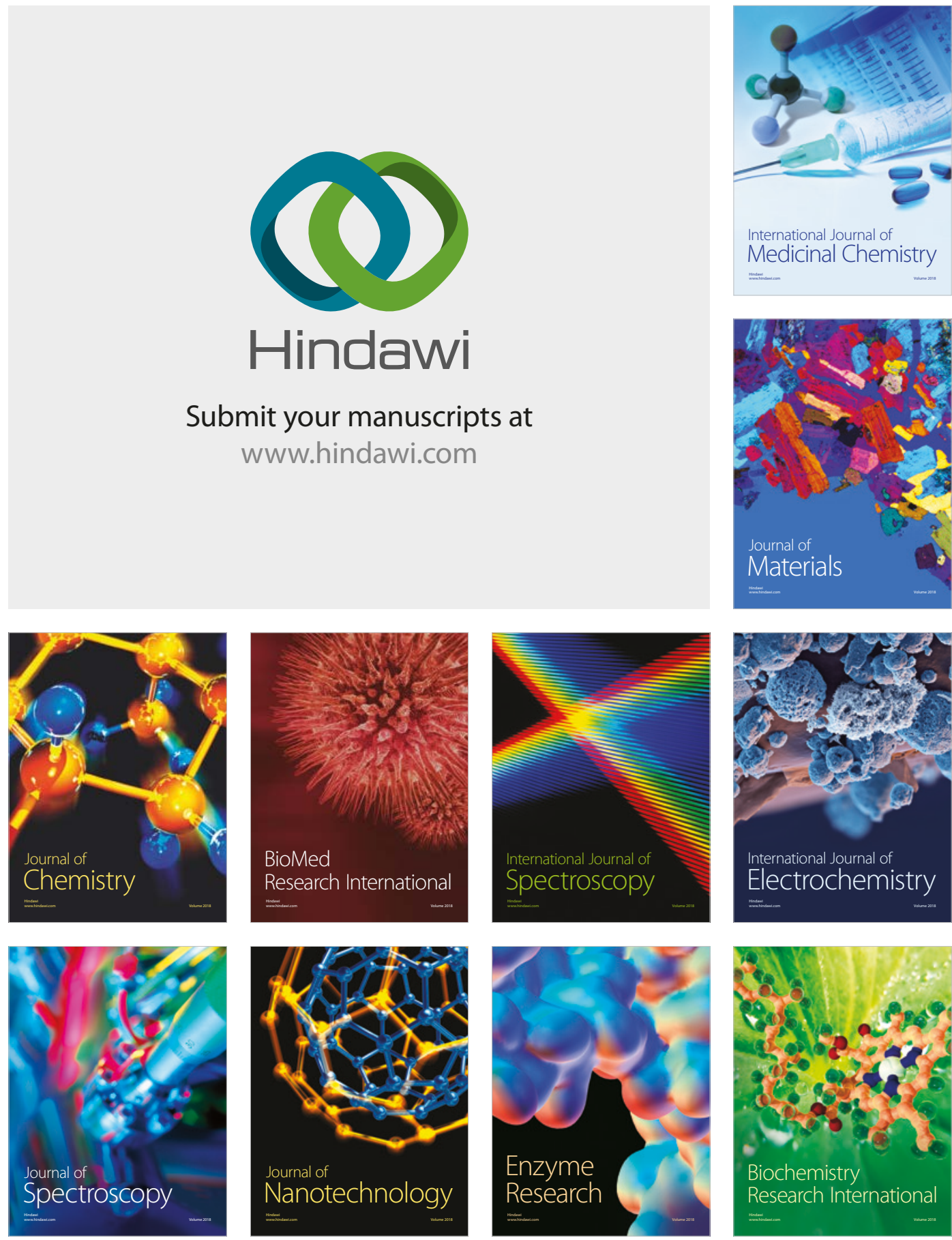
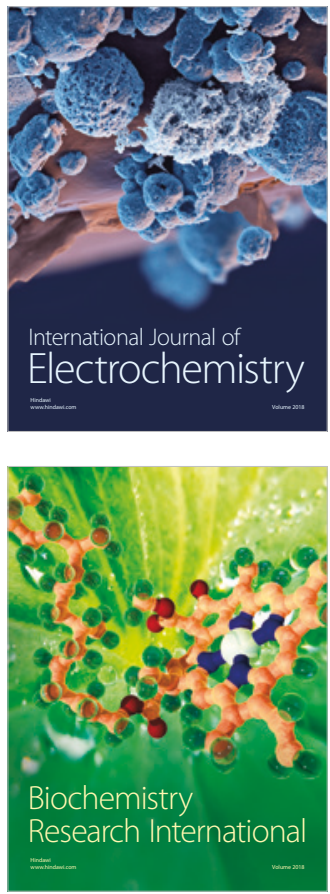